\title{
Oral Lichen Planus-A Challenging Case
}

\author{
Iquebal Hasan ${ }^{1 *}$, Rachel Lyon Beamer ${ }^{2}$ and Heather Moore Perry ${ }^{3}$ \\ ${ }^{1}$ Assistant Professor, Oral Medicine, ECU School of Dental Medicine, USA \\ ${ }^{2} D 3$ student, ECU School of Dental Medicine, USA \\ ${ }^{3}$ Dental Hygienist, ECU School of Dental Medicine, USA
}

*Corresponding author: Iquebal Hasan, Assistant Professor of Oral Medicine, ECU School of Dental Medicine, North Carolina, USA.

Received Date: October 22, 2019

Published Date: December 03, 2019

\section{Introduction}

Lichen Planus has been classified as both a cell-mediated immune response as well as an autoimmune condition. A target antigen in the reaction has not been identified. The mechanism of action and exact classification of the condition is not fully understood. Histologically, evidence of T-cell destruction of basal cells is observed. Tumor Necrosis Factor-Alpha (TNF-a) is thought to have major involvement in this reaction. Lichenoid reactions resemble Lichen Planus both clinically and histologically but differs due to evidence of local irritant or use of certain systemic medications. Local factors could be dental materials which could include but not be limited to gold crown, PFM crown, amalgam restorations and oral hygiene products [1]. Management of oral lichen planus and lichenoid reactions poses a challenge due to lack of full understanding of the etiology. Additionally, the multifactorial probable causes of these reactions contribute to the complexity of management [2]. Oftentimes, management of symptomatic lesions and trials of varying treatment options is necessary to determine effective modes of therapy for an individual patient.

\section{Aim}

The aim of this case report is to support the use of direct Kenalog injections in diabetic patients for successful resolution of Oral Lichen Planus if topical steroids do not work. In diabetic patients' systemic steroid is not an option because of the risk of spike in blood sugar level. Current accepted and researched modes of treatment include: Methotrexate $15 \mathrm{mg}$, Sulfasalazine, Clobetasol Propionate in aqueous solution and gel form, 1\% Pimecrolimun Cream, Hyaluronic Acid $0.2 \%$ Gel, Oral Alitretinoin 30mg, Levamisole Monotherapy 50mg, Topical Tacrolimus, Dexamethasone mouth wash, Photodynamic Therapy [3-13].

\section{Case Report}

An 82-year-old Caucasian female presented to East Carolina School of Dental Medicine in 2013 to establish comprehensive dental care. Patient reported history of lichen planus which was initially diagnosed approximately 40 years ago both clinically and through biopsy. Patient reported constant pain with lichen planus which worsened with certain foods; therefore, she was on a restricted diet. Patient's previous treatments included: Dexamethasone rinse, Lidex, alcohol-free Chlorhexidine, Clobetasol Gel, Kenalog Cream, Or a base, Imuran, Folic Acid, and systemic Prednisone. None of these treatments gave her substantial relief from pain except Prednisone. She stated that she had to stop taking Prednisone because it significantly raised her blood sugar level. She also informed that she has not been able to eat several things all these years and it has adversely impacted her quality of life.

After becoming a patient at East Carolina University School of Dental Medicine, she was initially treated with topical triamcinolone, which was ineffective. Following the ineffective treatment, the patient was recommended to consult with an Oral Medicine specialist at East Carolina University by her hygienist. During this evaluation, ulcerations surrounded by areas of erythema and reticular pattern were noted bilaterally in buccal mucosa, which aligned with existing crowns. Clinical presentation was characteristic of a lichenoid reaction. Patients medical history included Arthritis, Paget's Disease, Asthma, Hypertension, Diabetes Mellitus, and Heart disease. Two Recast infusions had been administered for Paget's disease, first dose was administered in 2007 and second infusion was administered March 2017. Medications she was taking are Vitamin D supplement, CoQ10, Folic Acid, 81mg Aspirin, Crestor, Levalbuterol Inhaler, Bystolic. Patient was allergic to Codeine, Demerol, Morphine. She denied any other allergy.

\section{Treatment}

After initial evaluation of the patient the Oral Medicine Specialist at ECU School of Dental Medicine decided on the line of treatment. Total of $4 \mathrm{mg}$ of Kenalog was injected directly into the affected 
areas, $2 \mathrm{mg}$ on each side and it was coupled with Clobetasol gel for home use. Patient was recommended to monitor her blood sugar every morning and evening till the follow up appointment. At the 2-week follow-up appointment, patient reported she experienced the maximum amount of relief she had in 40 years. Patient also informed that Kenalog did not adversely impact her blood sugar level. Patient was given same dose of Kenalog injection again and
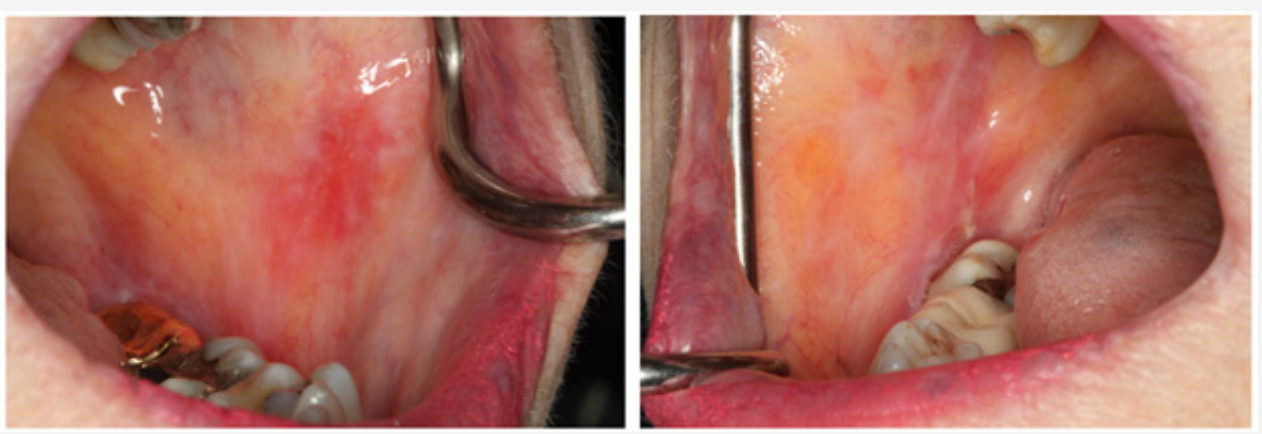

Figure 1: Pre-Treatment Photos.
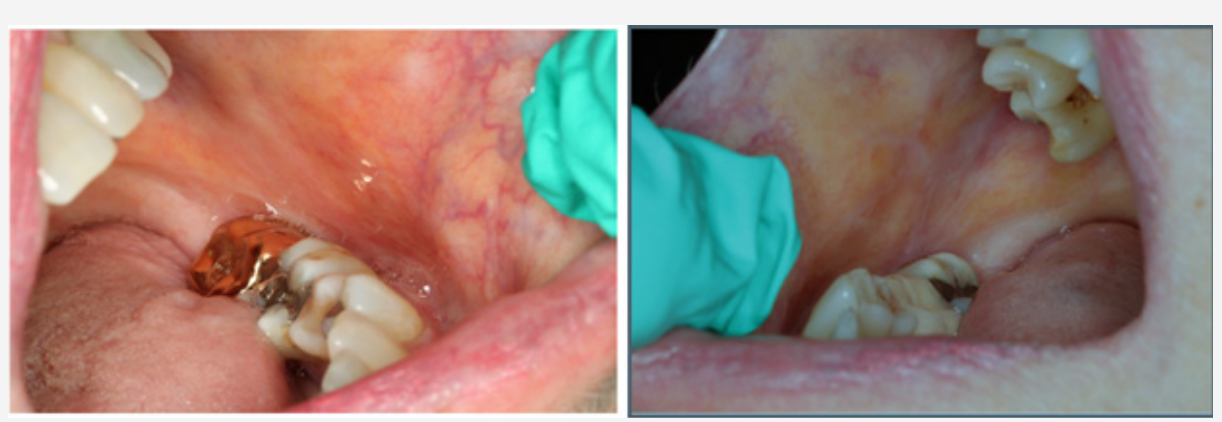

Figure 2: Post-Treatment Photos.

\section{Conclusion}

Occasionally lichen planus can be a treatment challenge. In some cases, patients do not respond to topical steroids and other conventional treatments. The use of systemic steroid in diabetic patients can have detrimental effects on blood glucose levels. In this case we saw that localized low dose Kenalog injections coupled with oral topical steroids can effectively treat lichen planus without effecting blood glucose levels. Further research needs to be conducted to establish it an accepted protocol in diabetic patients with persistent of oral lichen planus.

\section{Acknowledgement}

None.

\section{Conflict of Interest}

No conflict of interest.

\section{References}

1. Woo SB (2012) Oral Pathology: A Comprehensive Atlas and Text. Philadelphia: Elsevier Saunders.

2. Al Hashimi I, Schifter M, Lockhart PB, Wray D, Brennan M, et al. (2007) Oral lichen planus and oral lichenoid lesions: diagnostic and therapeutic considerations. Oral Surg Oral Med Oral Pathol Oral Radiol Endod 103: S25e1-S25e12. told to continue use of Clobetasol gel. In the next follow up in two weeks, full resolution of lesions and symptoms were noted. Patient reported she was able to eat foods without restrictions. She was told to stop use of Clobetasol. Patient was informed that she may have flare up of the lesion again. The next flare up was in four months but was less severe and it resolved with Clobetasol gel only (Figures 1\&2).

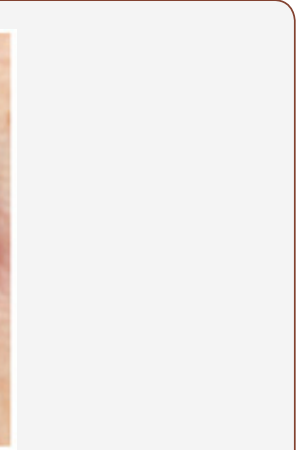


10. Won TH, Park SY, Kim BS, Seo PS, Park SD (2009) Levamisole Monotherapy for Oral Lichen Planus. Ann Dermatol 21(3): 250-254.

11. Ribero S, Stieger M, Quaglino P, Hongang T, Bornstein MM, et al. (2015) Efficacy of topical tacrolimus for oral lichen planus: real-life experience in a retrospective cohort of patients with a review of the literature. J Eur Acad Dermatolo Venereol 29(6): 1107-1113.

12. Bakhtiari S, Azari Marhabi S, Mojahedi SM, Namdari M, Rankohi ZE, et al. (2017) Comparing clinical effects of photodynamic therapy as a novel method with topical corticosteroid for treatment of Oral Lichen Planus. Photodiagnosis Photodyn Ther 20: 159-164.

13. Jajarm HH, Falaki F, Sanatkhani M, Ahmadzadeh M, Ahrari F, et al. (2015) A comparative study of toluidine blue-mediated photodynamic therapy versus topical corticosteroids in the treatment of erosive-atrophic oral lichen planus: A randomized clinical controlled trial. Lasers in Med Sci 30(5): 1475-1480. 